Original Research Paper

\title{
A Privacy Protected Platform to Aggrandize Micro-Business
}

\author{
Kaniz Fatema and M.M. Mahbubul Syeed \\ Professional Member, ACM (Association for Computing Machinery)
}

\author{
Article history \\ Received: 25-07-2019 \\ Revised: 01-10-2019 \\ Accepted: 07-11-2019 \\ Corresponding Author: \\ M.M. Mahbubul Syeed \\ Professional Member, ACM \\ (Association for Computing \\ Machinery) \\ Email: mahbubul.syeed@gmail.com
}

\begin{abstract}
This paper presents a platform, which will provide both the microbusinesses and its consumers a common ground to maximize their interests and benefits and in turn better contribute to the economy. This research work offers a comprehensive study on the micro businesses to portray their contributions and needs for expansion. The research then designs a conceptual platform to host, promote and manage businesses and their consumers. The platform is integrated with the MyGeoTrust privacy service for better protecting associated information. Finally, the research presents a prototype implementation of the platform in android to demonstrate its applicability.
\end{abstract}

Keywords: Micro-Business, Data Privacy, Geo Tagged Location Data, Virtual Business Platform Android, MyGeoTrust

\section{Introduction}

Micro-business often refers to the enterprises that are having less than ten employees, including the owner himself Pfitzner and McLaren (2018). Moreover, microbusiness served consumers within a given topographical location. Despite their size, such businesses collectively play a pivotal role within the overall economy of a country. For instance, the USA, the major economic powerhouse of the world, is enjoying 41.3 million jobs hosted by micro-businesses.

However, studies show that businesses of this scale often suffer from a comprehensive platform to publish and commercialize their business services, mainly due to budget constraints and workers Vega and Chiasson (2015). Therefore, a virtual platform equipped with an adequate feature set is an inevitable need for these businesses to effectively promote and mobilize their business services to a larger group of consumers with greater visibility.

Motivated by these factual needs, this research designed and implemented a platform to support micro businesses. The platform would offer a virtual mega mall with a versatile range of consumers. Each microbusiness will have the opportunity to leverage this platform to expose their business services to a larger community of consumers. For instance, through this platform a business can freely register within a category of business, can unveil their products and services, offers and promotions and can get the options to interact with customers (e.g., contacting, booking, buying, location services and others). Additionally, a business will also assess their business rivals and fix business strategies accordingly. This practice in turn will create a healthy competitive market with the best value-added services for the consumers Huda et al. (2019).

Another important aspect of the virtual platform is accessibility Deem and Brahnmath (2018). At any given time, consumers could effectively access to all the nearby business services, get their offers, compare among alternatives, favorite and $\log$ products and searches, subscribe for new promotions/offers, can do inapp communication and navigate themselves to the business places. In short, providing the virtual mega mall where they could get access to their desired services.

However, a platform of such purpose and scale often suffers from a lack of user data privacy and protection Zhang (2018). For instance, most of the major players on the track, including Google, Apple, Amazon and E-Bay collect and use users' personal data without their consent. They often use such data to capture better market share over their rivals. Nowadays, with growing concern, such access to personalized data is highly denounced by Internet users Yar (2018).

In this research, a virtual platform is proposed for a micro business that is directing to a single point references and get accessed to an attractive business service domain. In addition, the platform is integrating a state-of-the-art privacy protection service, named MyGeoTrust Guinness et al. (2015). We argued here that this integration would better protect users' data (for both business and consumers) from infringement and unauthorized access. To keep readers in sync, the mandate of MyGeoTrust project Guinness et al. (2015) is to offer a service that should provide privacy and transparency in crowd-sourcing user personal and location data as opposed to native platforms, e.g., Google. 
As, our proposed platform will use users' personal, business and location information to offer different services, therefore this integration with MyGeoTrust would achieve higher user confidence and use.

\section{Research Approach}

The key concern of this research is to offer a platform, as a pragmatic solution towards supporting a much-focused domain of business services (namely, micro-business) and their consumers. Alongside, the economic impact of this business segment and their needs must be documented. Accumulating these needs, this research adopted a Constructive Research Approach (CRA) and a Literature Review (SLR) approach.

Constructive Research Approach (CRA) dictates the methodology to offer innovative solutions to real-world problems Cornelissen et al. (2009), Rautiainen et al. (2017). CRA is thus a widely accepted research method in the domain of Computer Science and Software Engineering Piirainen and Gonzalez (2014). A perfect model of CRA should find a real-life problem requiring a pragmatic solution or get a thorough understanding of the problem domain. Design innovative artifact to solve the problem of understanding and offer corresponding implementation as a proof of concept. Finally, the CRA model contributes by carefully linking the solution to existing academic knowledge by investigative application scope.

The literature review is defined following the guidelines suggested in Kitchenham (2004) and the survey process used in Cornelissen et al. (2009). The step-by-step process is adopted for the review is adopted from Syeed et al. (2013).

The above two methodologies are utilized for this research under the following capacity,

The literature review is conducted to (a) aggregate the best available pieces of evidence in relation to the microbusiness ecosystem and their contribution to the overall economy of a country; (b) defines the requirements for microbusinesses to extend their business coverage and draw rationale in developing the proposed platform.

Thereafter, the CRA is practiced to (a) design the MVC based model for the platform, (b) integrate the data privacy service (MyGeoTrust) to ensure data security and privacy, (c) develop a working prototype to demonstrate the applicability of the platform and finally, (d) offer a comparative evaluation and assessment with the existing solutions to demonstrate the novelty.

\section{Results and Discussion}

\section{Review on Micro Business}

To trace down the contributions and needs of microbusinesses, a systematic review approach is carried out in this research. Micro-businesses have a significant contribution to the overall economy of a country. For instance, in the USA, micro firms represent $99.7 \%$ of all the employer firms Pfitzner and McLaren (2018). They pay $44 \%$ of the total payroll to more than $50 \%$ of employees in the private sector Vega and Chiasson (2015), Business (2013). More than 26 million direct jobs were hosted by the USA, while indirectly operating another 1.9 million jobs. Therefore, this business segment hosts 41.3 million jobs in the USA Huda et al. (2019).

In the USA, micro businesses created $64 \%$ of all the job opportunities during the last decade. Alongside, statistics reveals that business of this kind exhibits high survivability rate. For instance, a quarter of all micro businesses lasts for fifteen years or more, one third survives for twelve years, around $50 \%$ and $70 \%$ among these businesses survive for a minimum of five years and two years, respectively Vega and Chiasson (2015), Business (2013). These survival rates are comparable to major industries and states, which has similar survival rates.

In relation to high survivability, micro-businesses also show a growing trend for the last one decade. Despite economic depressions, many new businesses emerged with innovative and noble ideas and a large segment of them runs for a minimum of two years Vega and Chiasson (2015), Business (2013). Survival chances increase with the willingness to work hard.

These benevolent properties often inspire consumers and common people to vote in favor of micro businesses. As per statistics, in the USA, $90 \%$ consumers among all classes believe that such businesses have a long-lasting impact on the overall economy through job creation and taxation Pfitzner and McLaren (2018).

However, when it comes to promoting business services, it is observed that these businesses predominantly rely on online-based solutions. As shown in Fig. 1, 90\% of the means of business promotion relates to online services, including launching company website $(44.2 \%)$, email communication $(34.4 \%)$ and a promotional picture or video releasing on the website $(16.7 \%)$. The main objective behind such choice might be the cost-effectiveness of online services.

Furthermore, cheaper means are often chosen in creating and launching business websites. For instance, majority of the businesses $(82.2 \%)$ try to develop solution by themselves, or exploits cheaper alternatives, e.g., adopts readily available off-the-shelf software $(55.9 \%)$ or use basic editors $(35.7 \%)$ and templates (7.6\%) freely provided by the ISP. Table 1 offers a detailed listing of the alternatives popularly used in the USA.

These statistics and observations boldly indicate the fact that micro-businesses mostly use cheap but effective means to promote their services to reach consumers. Moreover, the most popular among them is online based services. Motivated by these facts, this research is committed to offering an online platform of benevolent features for both the micro-businesses and their consumers. 


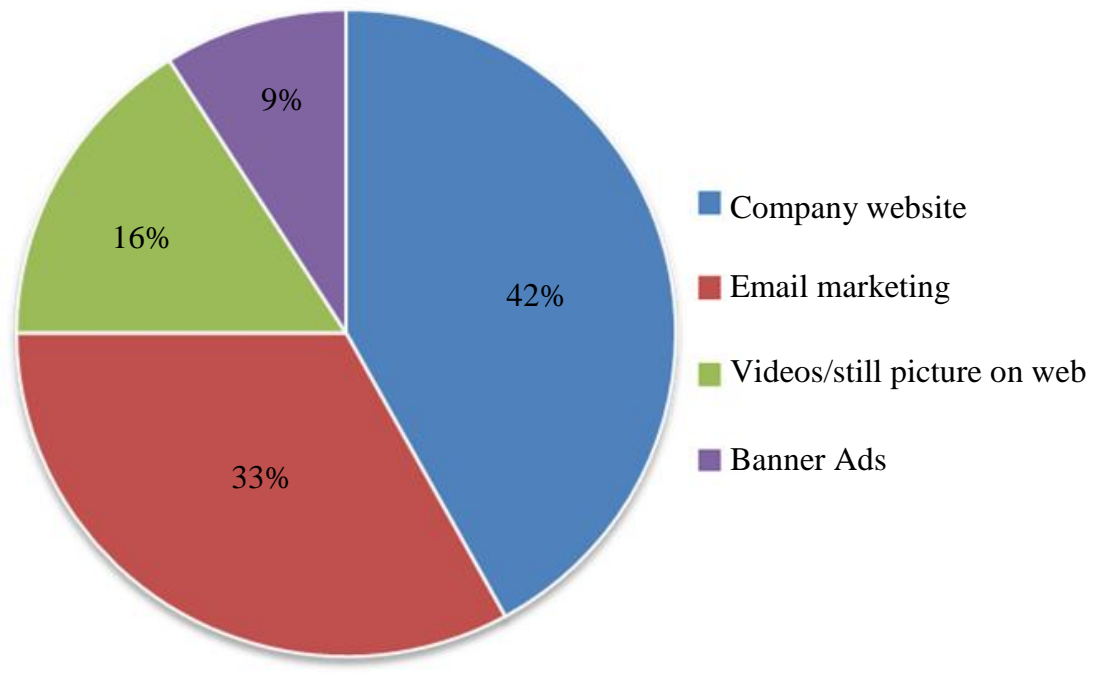

Fig. 1: Conventional method of small businesses promotion

Table 1: Small business website development methods

\begin{tabular}{ll}
\hline $82.2 \%$ & "Do It Yourself" (net) \\
$55.9 \%$ & Standard, off-the-shelf software for use on PC \\
$35.7 \%$ & HTML editor \\
$12.5 \%$ & Basic, templates provided by others \\
$7.6 \%$ & Basic, template provided by ISP \\
$2.1 \%$ & Browser-based, applications provided by ISP \\
& or others \\
$24.6 \%$ & "Do It for Me" (net) \\
$17.1 \%$ & Website, design firm/consultant \\
$5.0 \%$ & Friends/others \\
$3.0 \%$ & ISP or ISP, partners \\
$1.0 \%$ & The service provider that provided name \\
& registration and other services \\
\hline
\end{tabular}

\section{MyGeoTrust: The Privacy Platform}

The proposed platform is complemented by the integration of MyGeoTrust privacy service. This is mostly because user data of the proposed platform need to be protected as per user consent and priorities. This to note that the first two authors of this article were part of MyGeoTrust project and had contributed in terms of its design and development.

MyGeoTrust initiative is targeted to offer personal data protection (e.g., location information, browsing history, preferences) while individuals are using mobile applications that crowdsources personal data Guinness et al. (2015). Because, the current status quo offers very little control to the user over their personal data. The status quo on user data privacy reflects that the tech giants such as Google, Apple, Amazon often force users to accept a single point license agreement before proceeding with using their services. These license agreements in general offers the companies to collect, process, store and even share individual's personal usage data with third parties to gain market shares Fatema (2015).

However, privacy and security are increasingly becoming a major concern among mobile device users Kilintzis et al. (2014). A survey conducted on the US consumers concerning tracking and sharing on users, personal data by service providers showed that (a) $46 \%$ of the consumers fully condemn the sharing of personal data and (b) $92 \%$ respond that they don't prefer to receive tailored commercial adds based on their location information Cornelissen et al. (2009).

To address these concerns, MyGeoTrust project was launched in 2015 under the funding of the Finnish Funding Agency for Innovation. The project has been carried out in Finnish Geo Spatial Research Institute, Finland Guinness et al. (2015). The sole target of this project is to develop a platform for protecting user privacy in using geo-tagged, context-aware services. This platform will offer the users full autonomy, authority, transparency and control over their own personal data.

Current version of this project offers a client stack called MyGeoTrustClient that runs as a service in Android platform. The purpose of this stack is threefold: (a) allow users to track and control the usage and sharing of devices resources among third party applications, (b) allow user control over user personal data collection and usage by those applications and (c) implements a set of APIs' to create a standardize and traceable channel for these applications to use device resources.

The client stack is shown in Fig. 2 which is designed based on layered architectural pattern. First layer implements the configurable profiles to customize privacy settings for the applications 
running in users' device and the corresponding GUI. Through the GUI, users can create and edit profiles with different privacy settings and apply them on the third-party applications. The middle layer, i.e., the middleware stack implements two distinct modules: (a) the data crowdsourcing and privacy module and (b) the communication module. The earlier module collects user's data, such as Wi-Fi scans and sensor data, geo tagged location data and forwarded to the MyGeoTrust server.

However, this data collected, and storage is fully controlled and monitored by the user privacy settings. For instance, user can fully restrict to partially or fully allow user data collection and storage. Users can also verify, edit or remove their data collected by MyGeoTrust server. The later module (i.e., the communication module) implements the APIs through which the third-party applications gain access to the device resources. Most of these APIs mimics their Android counterpart in terms of service, however, they resource usage is controlled according to the privacy settings in the profile. For instance, an application can gain access to GPS for location information only if the user allows it through the currently active profile settings. This ensures that no application get explicit access to any device resources without users consent unlike native Android access privilege mechanism.

The third layer holds the native Android APIs that are wrapped by the MyGeoTrust APIs in the middle layer.

\section{The Platform for Micro-Businesses}

The proposed platform is designed and developed by leveraging client-server architectural pattern. As presented in Fig. 3, the left side details the android client application building blocks whereas the right side sketches the server.

The choice of a client-server architecture is evident as this platform requires cloud data storage to store, manipulate and access user's data through a client application.

\section{MyGeoTrustClient}

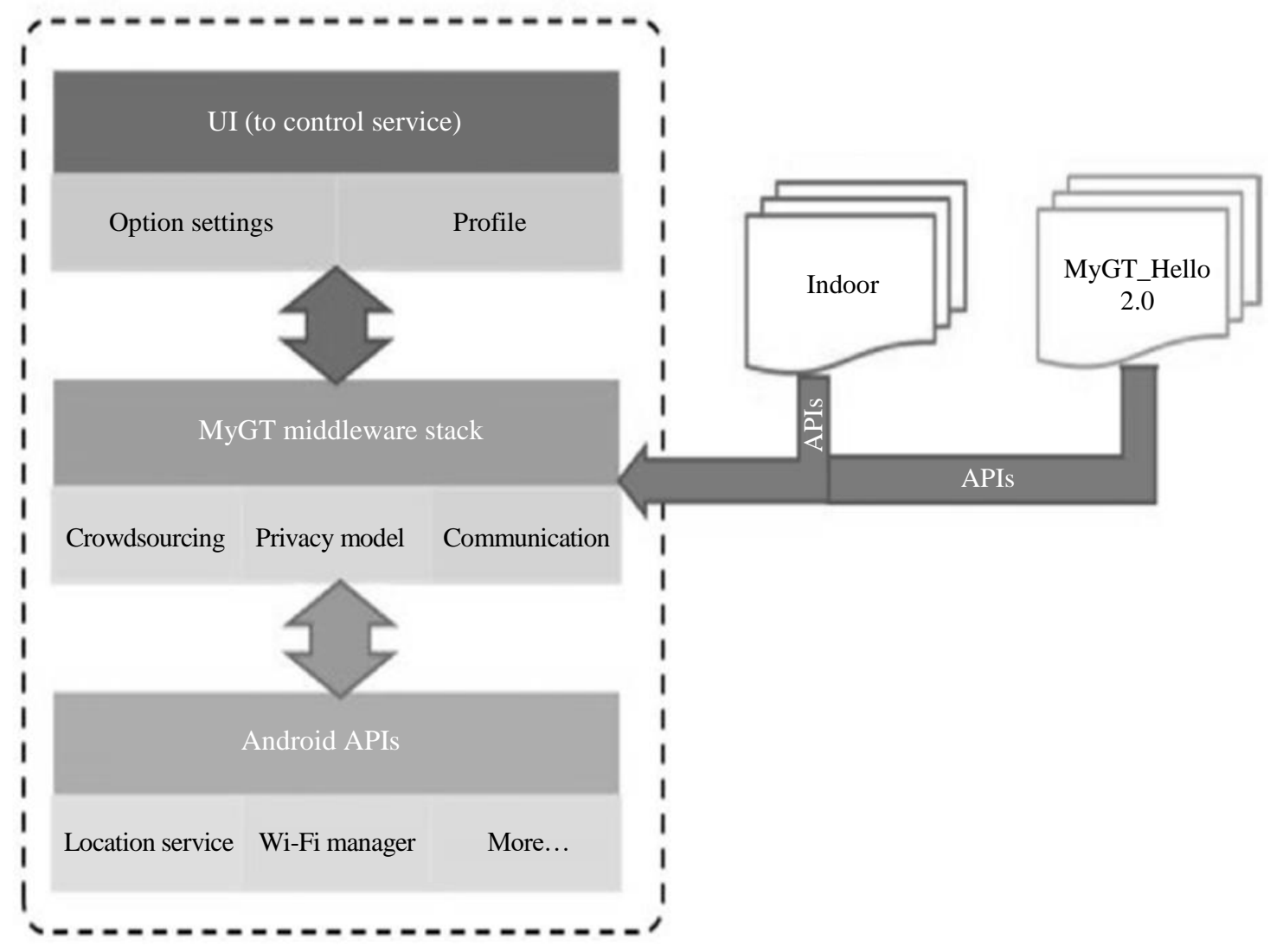

Fig. 2: MyGeoTrust client stack architecture Guinness et al. (2015) 


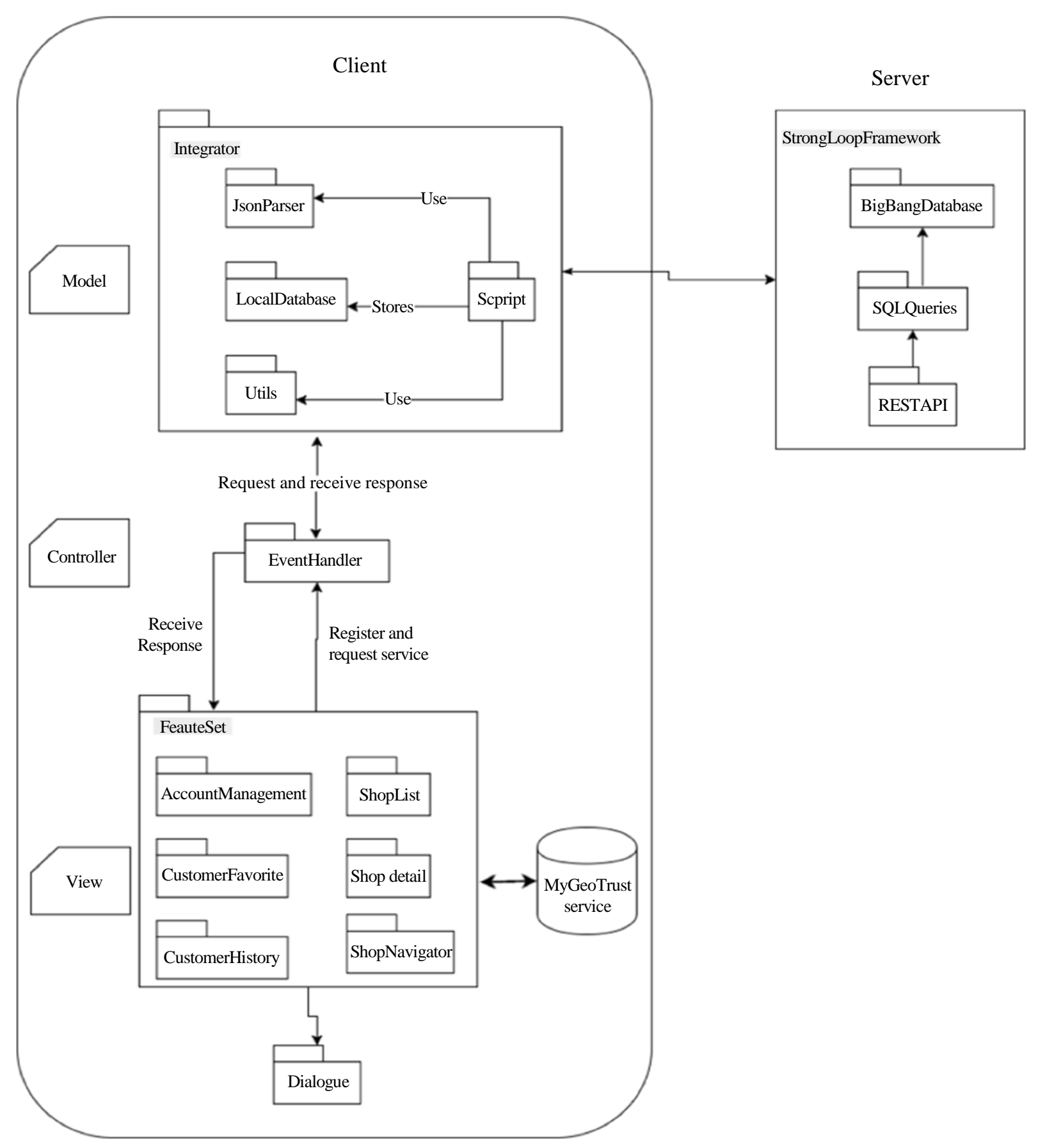

Fig. 3: The abstract architecture of the platform

The client is designed and implemented following Model-View-Controller (MVC) architectural pattern as can be seen from Fig. 3. MVC architectural pattern is the predominant choice for this application as it separates program logic from user interfaces, therefore, subsequently boosting performance and user experience.

Under this architecture, the Controller receives all the requests from client and process them using appropriate
Models and prepare data for the view. The View then generates final presentable response by using the prepared data sent by the controller.

Maintaining the mandate of MVC architecture, The Model (the top left part in Fig. 3) of this application handles all backend communications and data processing. This includes, (a) data exchange between client and the server using REST APIs; (b) parsing, 
processing, storage or update received data in the local database and (c) notify the controller to update corresponding View. These tasks are implemented in four distinct packages, namely, JsonParser, LocalDatabase, Scripts and Utils. The Scripts package, upon receiving request from the Controller

executes an asynchronous network call to the server using appropriate REST API. For instance, if the controller request for a list of shops under a certain category then a REST API with relevant parameters will be issued by the Scripts package to retrieve the data from the server. Upon receiving data in JSON format, the JSONParser package parse the data and stored in the LocalDatabase. While done, the controller notifies appropriate views to update.

The View (bottom part in Fig. 3) on the other hand implements all the GUI and the renders to present data for the user. Analogous to the Model, the View also consists of several packages responsible to perform specific functionalities. For instance, ShopList package renders shop lists under a selected category. The ShopDetail package mines and displays a shops' description, offers, associated communication and navigation options. The ShopNavigator package implements real-time, turn-by-turn navigation service for a selected shop. Finally, the CustomerHistory and CustomerFavorite packages implements bookmarking and browsing history management for each user. All these packages are kept under the FeatureList package and can be extended with additional features if required.

Alongside, user notification management is implemented in the Dialogue package that supports runtime customizable dialogue for graphical user notification. This package is used by all the packages listed under FeatureSet package.

The Controller (middle part in Fig. 3) is implemented in the EventHandlers package. The tasks of this controller include the followings: (a) upon receiving a request from a View it registers a callback, (b) hands over the request to appropriate Model to process, (c) upon receiving the result from the Model, it updates the requesting View with the received result. To put this discussion in perspective, consider that a user taps a shop in the shop list view. The view then registers a callback event to the Controller. The controller in turn hands over the request to the Model with shop name and id. While receiving detail of the requested shop, the Controller forwards the same to the ShopList view using the registered callback. The ShopDetail view is then opened with the retrieved shop detail.

To ensure data privacy and security, MyGeoTrust service need to be integrated with this platform. As shown in the bottom part of Fig. 3, this integration is done with FeatureList package. This arrangement is specific to Android applications due to their architecture.
E.g., each android application has a launching activity to start the application while clicked. For this platform, ShopList is that activity. Therefore, MyGeoTrust service is attached (in Android it's called Binding) this activity. While, ShopList is launched, it internally first Binds to the MyGeoTrust service and starts it if not already started. The service in response sends a binding reference to the ShopList activity. This reference is then used for further communication.

In current design and implementation, MyGeoTrust service is being used to protect users' geo-tagged location while using the in-app navigation service for navigating to a shop.

Real time turn-by-turn navigation service is implemented in the ShopNavigation package. While invoked, this package opens the navigation view and leverages Google map to plot the shop located on it. If turn-by-turn navigation is requested, then this navigation view will first register for continuous location update to the MyGeoTrust service. This service in response will allow access to its location update API to navigation view. This view will then use the API to provide navigation service, like google navigation (this arrangement is shown in Fig. 4). As this navigation feature is using MyGeoTrust service as opposed to Google native location service, therefore, user's location information will not be collected by Google without user consent.

The demo application named "BigBang" is implemented to test and verify the applicability of this proposed platform. As discussed, the client-side implementation is done in Android and for the server side, MySQL and StrongLoop framework are used.

The main motive of selecting Android is its huge market share at the present time. Android holds a staggering of $60.39 \%$ of the mobile operating system market, which make it an inevitable choice for this research Al Ameen et al. (2012). Additionally, MyGeoTrust privacy service is implemented in native Android, which makes the Android platform as an obvious choice.

The selection of MySQL for database implementation is mainly because it is an open-source RDBMS and the most widely used (second among all RDBMS) open-source client-server model RDBMS Müller et al. (2011). Additionally, it is highly reliable, high performing, scalable and cost-effective.

The StrongLoop framework is used as a middleware for this implementation. Contemporary application servers, databases, enterprise service buses (ESB) and mainframes are now using this framework for creating high quality, scalable and secure APIs. Recently IBM has procured this platform, recognizing its high market potential and pouring significant investment for its rapid development. 


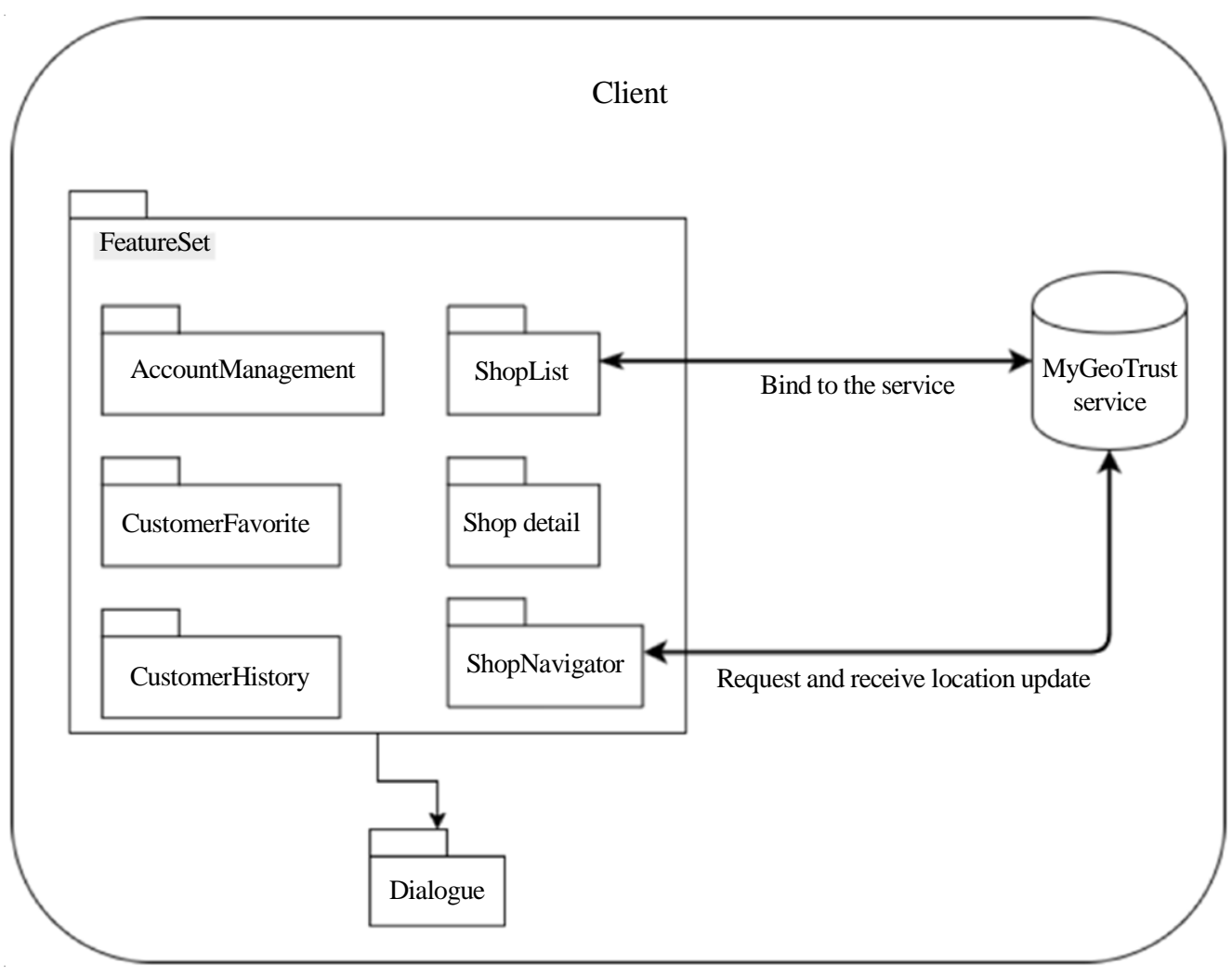

Fig. 4: Integration architecture with MyGeoTrust

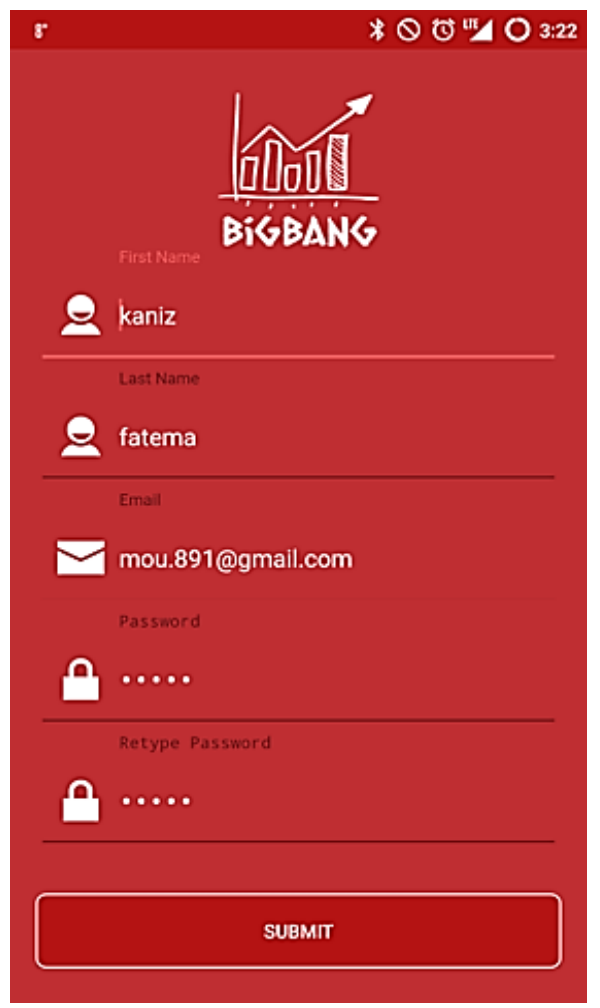

Fig. 5: User accounts create or manage the interface
Table 2: Third party libraries used

\begin{tabular}{ll}
\hline Library used & Purpose \\
\hline EventBus & $\begin{array}{l}\text { For registering callbacks and } \\
\text { event management. }\end{array}$ \\
EasyDialog & $\begin{array}{l}\text { For creating Custom dialogs to } \\
\text { user notifications and messages. } \\
\text { For efficient image rendering and } \\
\text { Pemory management. }\end{array}$ \\
Android Image Slider & For image display and sliding. \\
Fabmenu & $\begin{array}{l}\text { For creating animated floating } \\
\text { menu item. }\end{array}$ \\
\hline
\end{tabular}

Apart from the above-mentioned platforms, a set of open source libraries are also used for the development that is summarized in Table 2.

\section{BigBang Application: The Prototype Tool}

At the very first run, the "BigBang" application asks the user to register with an account (Fig. 5). Account registration for a user (both customers and businesses) is a mandatory precursor to use the platform. Because, customized services and features can only be provided with registered user information. For instance, a registered business can have its own business account where they can create customized business portfolio and promote business offerings. Alongside, businesses can communicate with registered users who request to 
receive notification from the businesses. For customers, they can create favorite listing of businesses, services, get notifications for business promotions or browse their history among others.

Successful registration of an account allows a user to use the platform with that account as default. All options (e.g., business categories, favorite list, browsing history) are loaded in a Navigation drawer against the account (Fig. 6a). Furthermore, users can create a new account, manage/update an existing account or switch between accounts through this navigation drawer, as shown in Fig. 6b. User's personalized information is updated with the switching of the user account on this interface. Users can access all shops/businesses under a business category (e.g., Fashion and beauty) by tapping it in the navigation drawer (Fig. 6a).

The list of shops/businesses under a selected category is shown in a list view (Fig. 7a). Tapping on a shop in this list opens the shops' personalized page (Fig. 7b). This page details business information that includes, shops promotional picture gallery, its' portfolio, shops business details (e.g., full product listing, website address, phone number, email and so on), auto calculation of shops' business hours and updating users.
Additionally, shops' personalized page provides customers in-app communication services in five distinct ways, e.g., (a) make a phone call to the shop, (b) email, (c) browse shops personal website, (d) add the shop in users favorite list to get promotional notifications and (e) use in build navigation service for navigating to the shop. All these features are implemented using selfdescriptive floating buttons (Fig. 7b). A demo of a phone call is presented in Fig. 8a.

To use the turn-by-turn navigation service, users need to tap the navigation floating button in a shops' personalized page (the rightmost button with a map icon in Fig. 7b). As a result, the navigation window will be opened (Fig. 8b) and plot the shop's location as a bubble marker on google map and lists navigation options (e.g., get the optimal driving route from the users' current location to the shop, start real-time turn-by-turn navigation) as shown in Fig. 9. For showing the route, the platform first acquires users current Geo location and then retrieves optimal route to the shop from google map service. Upon receiving the route information, it is plotted on the map (Fig. 9a). It also displays the total distance and the required time to travel on the top right corner (Fig. 9a).

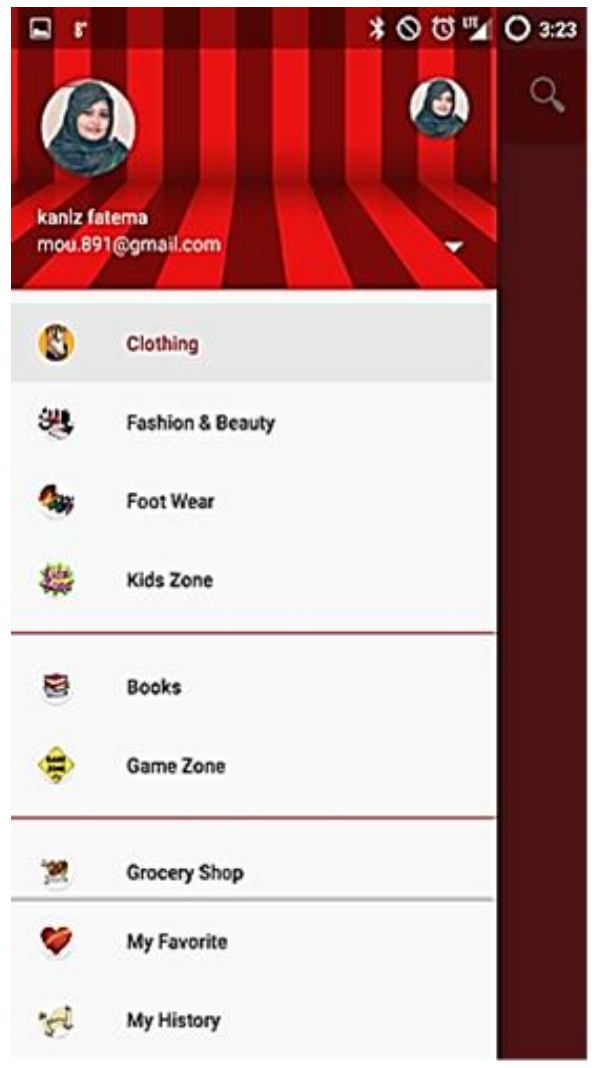

(a)

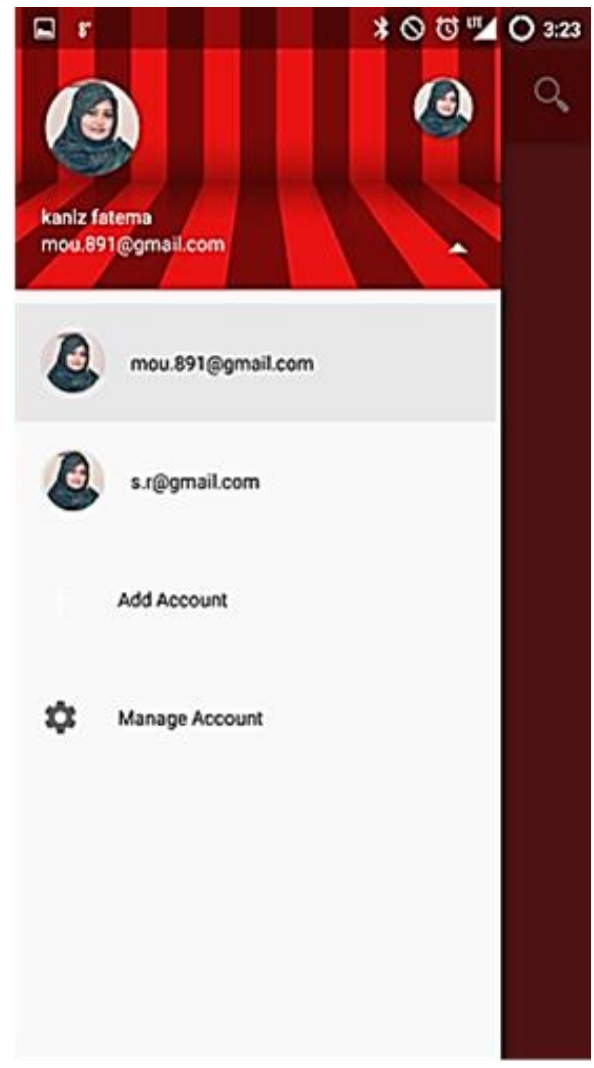

(b)

Fig. 6: (a) Services offered by the Platform is listed under the user account, (b) Management of User accounts 

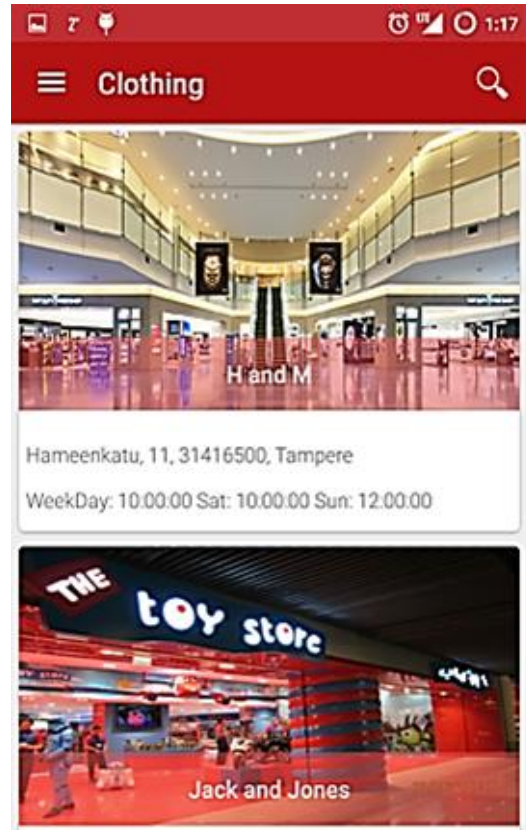

Hirvisuontie, 3, 2810, Espoc

WeekDay. 10.00 .00 Sat: 09.00 .00 Sun: 12.00 .00

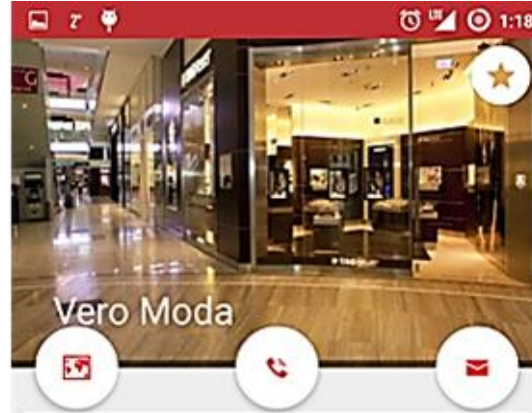

Shop short description goes here. This shop is an exicting

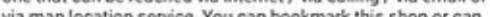
viem plocation senice. You can tookmark this shop or can service and this app is amaizing

Shop is now open/close

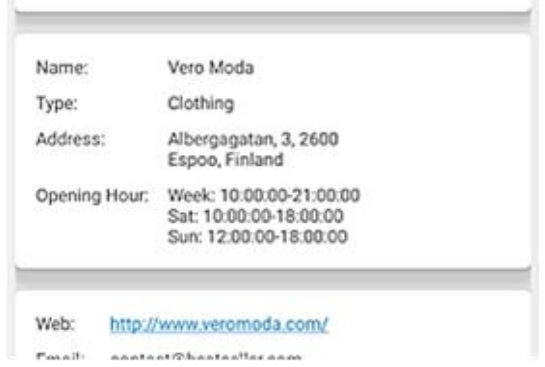

(b)

Fig. 7: (a) A list of all shops/businesses under the category "Clothing”, (b) A shop/business personal page

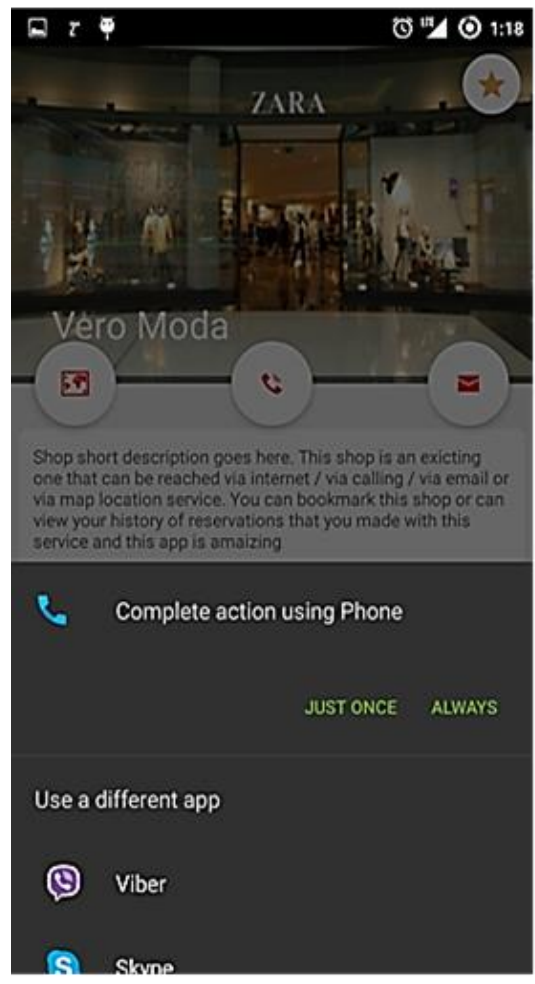

(a)

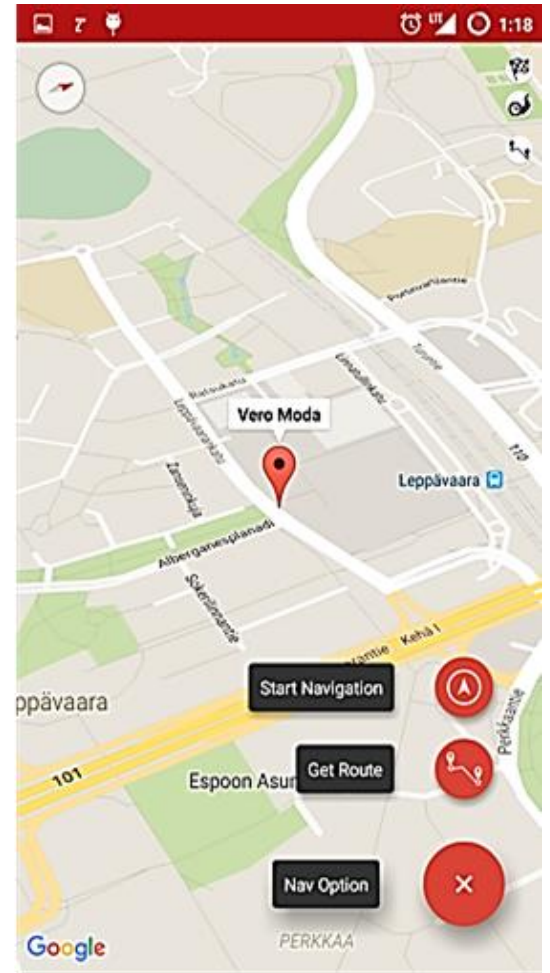

(b)

Fig. 8: (a) In-App phone communication, (b) Navigation window plotting the shops' location on the google map with available navigation options 
Kaniz Fatema and M.M. Mahbubul Syeed / Journal of Computer Science 2019, 15 (11): 1595.1606 DOI: $10.3844 /$ jessp.2019.1595.1606

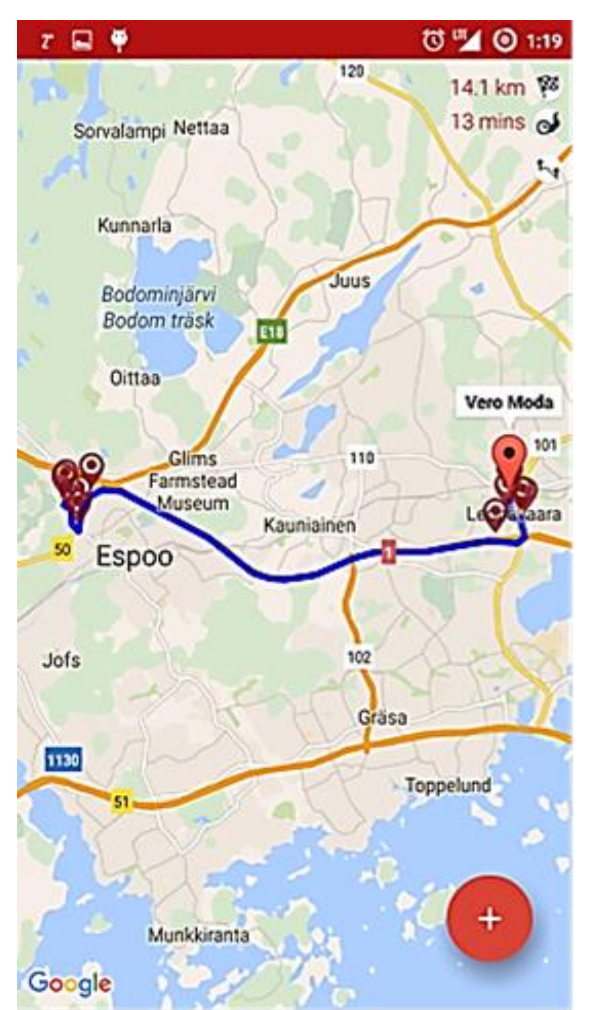

(a)

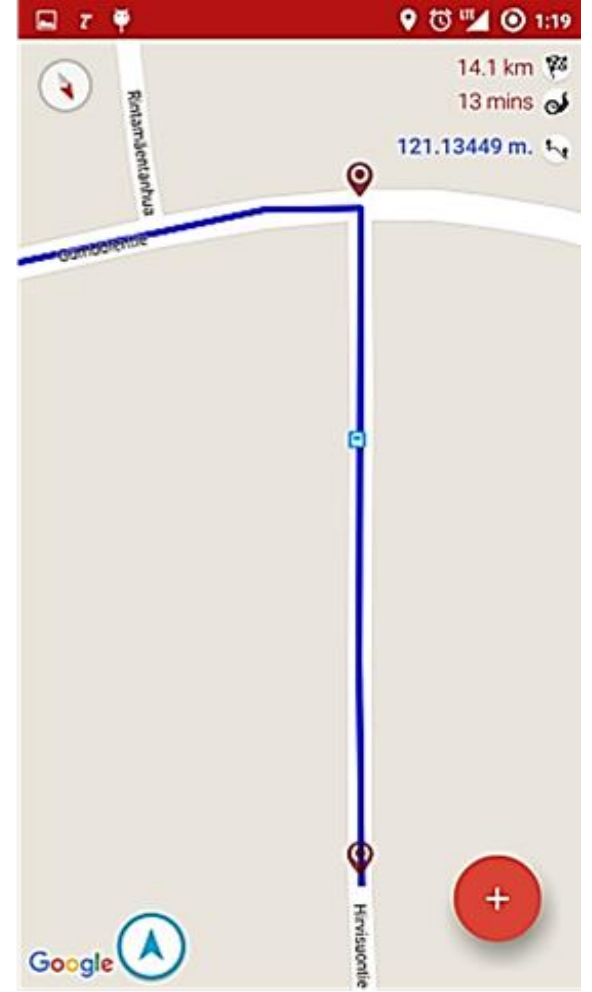

(b)

Fig. 9: (a) Plotting the optimal driving route between the user and the shop, (b) Starts real-time turn-by-turn navigation service

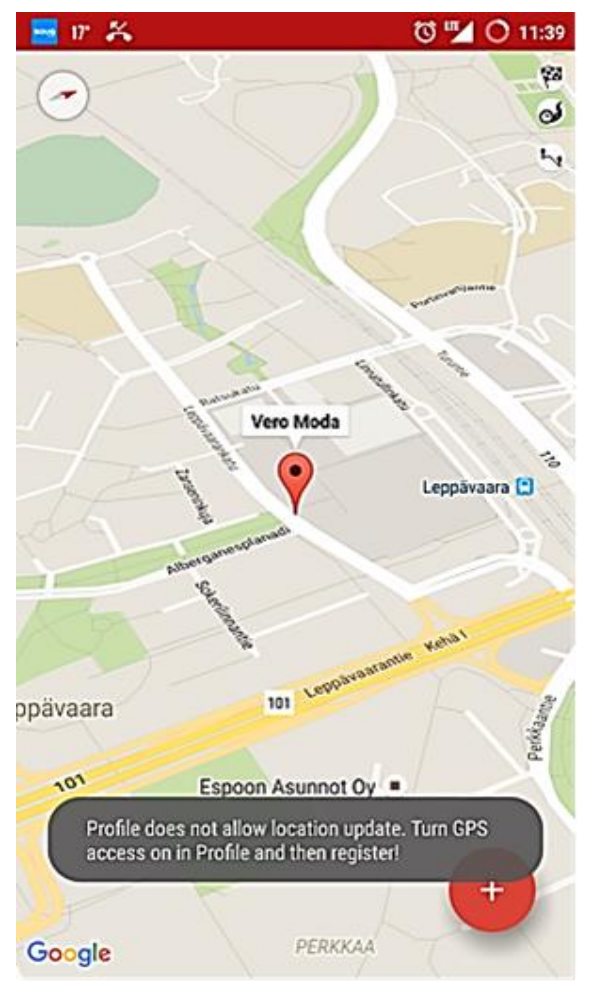

(a)

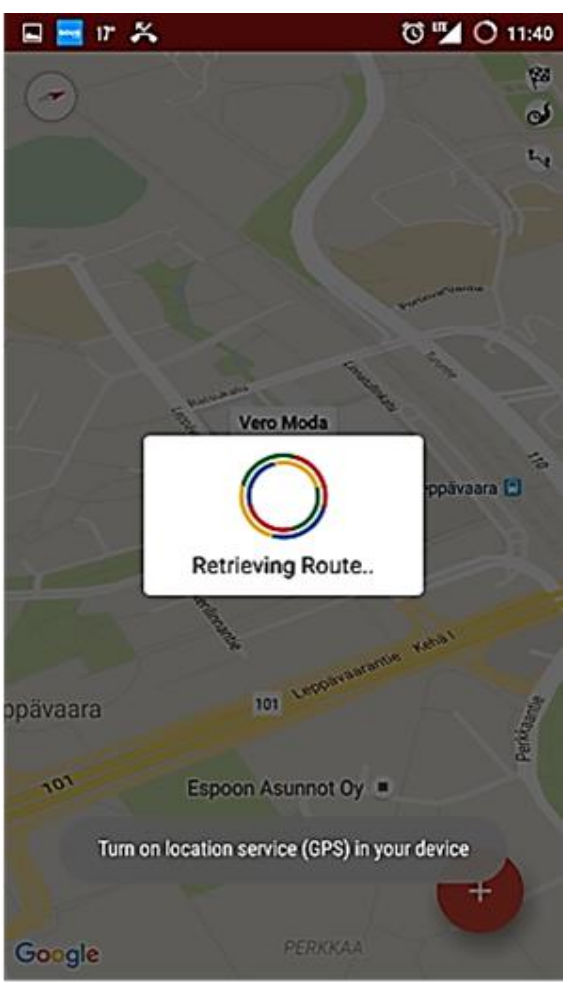

(b)

Fig. 10: (a) Location Service is prohibited by MyGeoTrust user setting, (b) GPS is not currently turned on in the device 
The navigation service can be initiated (Fig. 9b) by tapping the Start Navigation menu option (in Fig. 8b). During this navigation, user's current location is plotted over the route by taking continuous Geo location update (the arrow location marker in Fig. 9b). While the location marker approaches towards a turn in the route (e.g., within $20 \mathrm{~m}$ of the next turn), the map rearranges to show the next turn. The distance between location marker and the next turn is updated continuously and are shown on the top right corner of the screen (Fig. 9b). The navigation stops if the destination is reached or the user stops the navigations service.

A fully customized user notification and messaging service is implemented within this platform to appropriately guide the users. E.g., messages regarding the current privacy and device settings are displayed to guide the users. As shown in Fig. 10a and 10b, while using the navigation service, the platform checks whether the MyGeoTrust privacy settings allow location update and whether the GPS is currently turned on or not in the device, respectively and notify user with appropriate messages.

\section{Validation}

Existing platforms sharing similar concept and services (e.g., Amazon (2019), eBay (2019), Alibaba (2019)), are targeted for global scale and are designed to fit for all. While such approaches have many benefits, they often fall short in ensuring the need for microbusinesses. For instance, micro-businesses like a saloon, pizza shops, food courts, stationery shops etc. often focus on consumers at their vicinity and operate locally.

Additionally, they often cannot launch and promote their own web platform and are not targeting the global market. Likewise, consumers who are looking for such services in near proximity cannot effectively trace them due to lack of a comprehensive web platform. Therefore, the problem targeted for this research is pragmatic and relevant one.

Moreover, for all the existing platforms (as listed above) user data privacy are solely on the hands of the platform owner and user must agree on the terms and conditions to avail their services. In contrast to this status quo, our platform offers a transparent tracing and control of user data access, storage and processes. Therefore, ensuring personal and business data privacy and security.

However, user experience validation has not been conducted with the prototype and is listed as a future extension of the work.

\section{Conclusion and Future Work}

This research has conducted a review on microbusiness requirements. After that, the research proposed a platform for micro business as well as implemented the platform, which is targeted to support micro-businesses and their consumer base. This platform should offer a single point reference of interaction between businesses and their consumers. The research work explores the opportunity to integrate the MyGeoTrust privacy service with the proposed platform to provide users a secure and trustworthy means in using online shopping and data exchange, specifically, for location-based services, e.g., the turnby-turn navigation service.

We also argued here that this solution would lead to a sustainable and healthy business ecosystem for micro businesses. Because, each business can advertise their services with an opportunity to compare with the rivals to provide better offers. This may initiate a competitive market with the maximization of value-added services for the consumers. Moreover, due to the availability of detersive services within a unified platform, each business can reach out to a large consumer base, which is otherwise, would not be possible. On the other hand, consumers will get a virtual mega mall of all local services located around their vicinity.

\section{Author's Contributions}

Kaniz Fatema: This work has been accomplished as a M.Sc thesis of this author. She is the core contributor of this work.

Mahbubul Syeed: This author was one of the supervisors of the thesis and work extensively towards preparing the article.

\section{Ethics}

This article is original and contains unpublished material. The corresponding author confirms that all of the other authors have read and approved the manuscript and no ethical issues involved.

\section{References}

Al Ameen, M., J. Liu and K. Kwak, 2012. Security and privacy issues in wireless sensor networks for healthcare applications. J. Med. Syst., 36: 93-101. DOI: $10.1007 / \mathrm{s} 10916-010-9449-4$

Alibaba, 2019. Available at www.alibaba.com

Amazon, 2019. Available at www.amazon.com

Business, 2013. Micro business statistics and trends.

Cornelissen, B., A. Zaidman, A. van Deursen, L. Moonen and R. Koschke, 2009. A systematic survey of program comprehension through dynamic analysis. IEEE Trans. Software Eng., 35: 684-702. DOI: $10.1109 /$ TSE.2009.28

Deem and Brahnmath, 2018. Amazon Technologies, Inc., assignee. Virtual world electronic commerce platform. United States Patent Application US $14 / 575,840$. 
eBay, 2019. Available at www.ebay.com

Fatema, K., 2015. BigBang: An application towards building a business ecosystem through the integration of MyGeoTrust privacy platform. MSc Thesis.

Guinness, R., H. Kuusniemi, Vallet, Sarjakoski and J. Oksanen et al., 2015. MyGeoTrust: A platform for trusted crowdsourced geospatial data. Proceedings of the 28th International Technical Meeting of the Satellite Division of the Institute of Navigation, (DIN' 15), At Tampa, Florida, USA.

Huda, M., S.L. Qodriah, B. Rismayadi, A. Hananto and E.N. Kardiyati et al., 2019. Towards Cooperative with Competitive Alliance: Insights into Performance Value in Social Entrepreneurship. In: Creating Business Value and Competitive Advantage with Social Entrepreneurship, Iyigun, N.O. (Ed.), IGI Global, ISBN-10: 1522556877, pp: 294-317.

Kilintzis, V., N. Beredimas and I. Chouvarda, 2014. Evaluation of the performance of open-source RDBMS and triplestores for storing medical data over a web service. Proceedings of the 36th Annual International Conference of the IEEE Engineering in Medicine and Biology Society, Aug 26-30, IEEE Xplore Press, Chicago, IL, USA, pp: 4499-4502. DOI: 10.1109/EMBC.2014.6944623

Kitchenham, 2004. Procedures for performing systematic reviews. Technical Report TR/SE-0401, Keele University and Technical Report 0400011T.1, National ICT Australia.

Müller, R.M., B. Kijl and J.K.J. Martens, 2011. A comparison of inter-organizational business models of mobile app stores: There is more than open Vs. closed. J. Theoretical Applied Electronic Commerce Res., 6: 63-76.
Pfitzner, D.M. and J. McLaren, 2018. Microbusinesses in Australia: A robust definition. Australasian Account. Bus. Finance J., 12: 4-18. DOI: $10.14453 /$ aabfj.v12i3.2

Piirainen, K.A. and R.A. Gonzalez, 2014. Constructive synergy in design science research: A comparative analysis of design science research and the constructive research approach. Liiketaloudellinen Aikakauskirja, 3: 206-34.

Rautiainen, A., K. Sippola and T. Mättö, 2017. Perspectives on relevance: The relevance test in the constructive research approach. Manage. Account. Res., 34: 19-29.

DOI: $10.1016 /$ j.mar.2016.07.001

Syeed, M., Hammouda, I. and Systa, T., 2013. The evolution of open source software projects: A systematic literature review. J. Software, 8: 2815-2829. DOI: 10.4304/jsw.8.11.2815-2829

Vega, A. and M. Chiasson, 2015. Towards a comprehensive framework for the evaluation of small and medium enterprise policy. Evaluation, 21: 359-75. DOI: 10.1177/1356389015593357

Yar, M., 2018. A failure to regulate? The demands and dilemmas of tackling illegal content and behaviour on social media. Int. J. Cybersecurity Intell. Cybercrime, 1: 5-20.

Zhang, D., 2018. Big data security and privacy protection. Proceedings of the 8th International Conference on Management and Computer Science (MCS’ 18), Atlantis Press. 